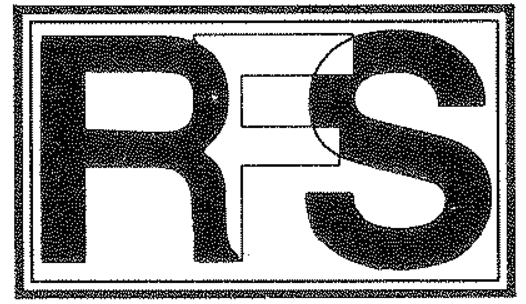

Revista de Fomento Social, 49 (1994), 583-598

\title{
La reforma del mercado de trabajo y el nuevo marco jurídico-laboral
}

La autora de este trabajo, desde una perspectiva crítica con la reciente reforma laboral, analiza el nuevo panorama normativo surgido a raíz de la misma, distinguiendo las reformas operadas de las proyectadas. Estudia de modo sucinto las reformas realizadas que afectan a los mecanismos de flexibilidad en la entrada al mercado de trabajo (régimen de colocación o modalidades de contratación) y a la flexibilidad en el desarrollo y extinción de la relación laboral (tiempos de trabajo, movilidad funcional y geográfica, despidos disciplinarios y por causas económicas, etc.). Este articulo contribuirá,junto con eltrabajo del profesor Alemán Páez que también publicamos en este número, a que nuestros lectores puedan tener conocimiento tanto del alcance concreto de las reformas realizada como de sus posibles consecuencias en la propia disciplina jurídico-laboral.

(*) Profesora de Derecho del Trabajo. Universidad de Granada. 


\section{Consideraciones previas}

La amplitud del tema que nos ocupa (1) nos obliga a realizar algunas consideraciones previas dirigidas a delimitar adecuadamente el objeto del presente artículo, así como la metodología con la que se aborda.

En primer lugar, se ha de advertir que se trata de un análisis predominantemente político-jurídico: aunque se han tenido en cuenta los aspectos economicos del tema, no se pretende aquí realizar un estudio interdisciplinar propiamente dicho. Aún con las limitaciones que esto implica, considero que es precisamente ésta, la perspectiva jurídica, la que como iuslaboralista me corresponde adoptar.

La reforma del mercado de trabajo es un tema que ha acaparado la atención de la opinión pública de nuestro país, más allá de los foros políticos, académicos y de aquéllos que se dedican profesionalmente a las relaciones laborales; sin embargo, el hecho de que las reformas legislativas hayan trascendido a la opinión pública no significa que se tenga un conocimiento exacto del alcance de la reforma operada y sobre todo de cuáles son los aspectos concretamente modificados, ya que se han ido sucediendo reformas parciales, que modificaban a las anteriores, y constantes enmiendas de los proyectos legislativos dados a conocer. El objeto de este trabajo es precisamente dar una vision del nuevo panorama normativo, distinguiendo las reformas operadas de las proyectadas e informando sucintamente del contenido de aquéllas.

En segundo lugar, también se han de hacer precisiones terminologicas, referidas a la no pormás extendida imprecisa expresión "reforma del mercado de trabajo". Por un lado, "mercado" es una institución económica -no en vano se trata de una reforma auspiciada por economistas y desde la economía-: por tanto, no parece este término el más adecuado para aludir simplemente a una reforma legislativa, ya que por más que ésta incida sobre el juego de oferta y demanda de empleo o sobre el intercambio de trabajo por salario, en el mismo confluyen numerosas variables económicas, demográficas, educativas, sociológicas y jurídicas no laborales -aspectos fiscales, administrativos y mercantiles- que no han sido acometidas en esta denominada "reforma del mercado de trabajo". Con ésta

(1) El presente trabajo tiene su origen en una conferencia pronunciada en el Centro Universitario Francisco Suárez el 3 de marzo de 1994 cuyo título "La reforma del mercado laboral" ha sido oportunamente modificado para su actualización a las reformas legislativas que efectivamente se han consumado, fundamentalmente, la Ley 11/1994, de 19 de mayo. 
sólo se alude a una reforma de la normativa laboral, por lo cual se opera un reduccionismo que, además, encierra connotaciones culpabilizadoras del Derecho del Trabajo en cuanto encierra una doble premisa: que el desempleo viene provocado por el marco jurídico-laboral (por su rigidez) y que modificando dicho marco se conseguirá elevar la tasa de ocupación. Dichas premisas habrian de verse confirmadas a partir de la entrada en vigor de la Ley 11/1984, de 19 de mayo, por la que se modifican determinados artículos del Estatuto de los Trabajadores y del Texto Articulado de la Ley de Procedimiento Laboral y de la Ley de Infracciones y Sanciones en el Orden Social. La finalidad de esta Ley, además de potenciar el papel regulador de la negociación colectiva (aligerando el acervo de normas mínimas estatales de derecho necesario esperando que aquella sea "un instrumento fundamental para la deseable adaptabilidad") es, tal como queda expresado en la propia Exposición de Motivos, "incidir en los aspectos relacionados con una gestión más flexible de los recursos humanos en la empresa, en temas como la ordenación del tiempo de trabajo y del salario, o las modificaciones en la prestación laboral, por considerar que una actuación en estos términos puede ser en muchos casos un mecanismo preventivo frente al riesgo de pérdida de empleo". En definitiva, la reforma arranca del diagnóstico avanzado por el Libro Blanco del crecimiento, la competitividad y el empleo, según el cual la salida de la recesión pasa necesariamente por una reducción del coste laboral y de las prestaciones sociales, como única manera de crear empleo y garantizar la competitividad de las empresas.

Si la competitividad y el empleo son los objetivos de todas las reformas operadas a finales de 1993 y 1994, el instrumento que se adopta no es otro que la flexibilización del marco jurídico-laboral mediante un procesode desregulación y de individualización de las relaciones laborales. Su resultado no será una mera reforma del sistema anterior, sino la instauración de un nuevo marco jurídicolaboral basado sobre nuevos principios.

Finalmente, para cerrar este apartado de consideraciones previas, y antes de entrar en la exposición de las principales modificaciones, es interesante destacar, aunque sólo sea de paso, dos variantes que han acompañado todo el proceso. Por un lado, el pacto social, el diálogo con los interlocutores sociales, como base previa a la actuacion legislativa: en realidad ha fracasado, tanto por la vía del Consejo Económico y Social -cuya intervención no vinculante está prevista en la Ley 21/1992-, como por la concertación social directa con los sindicatos, lo cual genera un clima social muy distinto al de la Ley del Estatuto 
de los Trabajadores de 1980, considerada como una verdadera "ley negociada".

Por otro lado, es difícil dejar de mencionar el Derecho Comunitario y el derecho comparado como supuestos argumentos legitimadores de la reforma, tal como de hecho aparecen esgrimidos en la Exposición de Motivos de la Ley 11/1994. Ciertamente, los problemas del incremento de las tasas de desempleo y las dificultades de las empresas para adaptarse a las nuevas condiciones del mercado que se plantean en nuestro sistema de relaciones laborales se detectan también en los países de nuestro entomo: por ello, prácticamente en todos los países que nos sirven de referencia se plantea la necesidad de introducir modificaciones en la ordenación del mercado de trabajo y, más concretamente, del marco institucional de las relaciones laborales. En el ámbito comunitario, el Libro Blanco sobre el crecimiento, la competitividad y el empleo pone de manifiesto la necesidad de relanzar la competitividad de las empresas europeas así como de combatir el desempleo; no obstante, este documento, más que aportar soluciones, no hace sino apuntar líneas o sugerencias muy generales tales como la mejora de los sistemas de formación profesional, la reducción de tiempos de trabajo y reparto del empleo, la vinculacion de los salarios a la productividad, la mayor facilidad a la movilidad funcional y la reducción de la presion fiscal y de los costes de seguridad social.

La reforma operada en nuestro país alcanza dimensiones muy superiores a las acaecidas en otros estados de la Unión Europea y ello tanto cuantitativamente (por el mayor número de instituciones afectadas) como cualitativamente (por la profundidad de los cambios). Ciertamente, en distintos Estados europeos se han sucedido reformas puntuales de instituciones laborales, pero ello no ha de llevar a la idea de que se ha producido un cambio unívoco o unidireccional; tampoco se puede hacer un juicio sobre la mayor o menor rigidez de nuestro sistema en comparación con el resto amparándose en la confrontación de elementos aislados o secundarios, ya que cada pieza juega un papel diferente según el sistema en el que se inserte. Por tanto, la supuesta adaptación comunitaria es un argumento que pertenece más al terreno de la confrontación ideologica que al puro debate jurídico, por más que se haya tratado de reconducir a este último campo.

Adicionalmente, en el terreno del Derecho Comunitario, no puede afirmarse, si exceptuamos la normativa sobre tiempos de trabajo o de seguridad e higiene (materia ésta cuya reforma ha sido pospuesta), que se haya producido un conjunto de disposiciones a las que supuestamente habría que adaptarse. 


\section{Breve referencia al proceso de la reforma, su justificación y límites}

A los efectos de poder llegar a una valoración panorámica de la modificación del marco institucional de las relaciones laborales es imprescindibie hacer una reflexión evolutiva del mismo, limitándonos, desde luego, a la etapa más reciente.

El sistema de relaciones laborales surgido a partir de la Constitución de 1978, su marco institucional, se relaciona ya con la idea de crisis. De hecho, el Estatuto de los Trabajadores (LET) represento una flexibilización muy importante respecto al sistema anterior, línea que continuó en las sucesivas reformas de la LET; por tanto, no puede afirmarse que la inspiración de esta reforma sea del todo nueva. La novedad reside en los instrumentos utilizados y en el alcance de la misma, suponiendo la instauración de un nuevo sistema que modifica sensiblemente al instaurado a partir de la democratización de las relaciones laborales en nuestro país.

Tras la firma del Tratado de la Unión Europea el Gobierno presentó el Programa de Convergencia para elevarla competitividad de las empresas, afectando también a las relaciones laborales (se refería a la necesidad de flexibilizar el mercado de trabajo, a la moderación salarial, la agilización de la colocación, la reforma del INEM, la eliminación de límites a la movilidad fun-cional y geográfica.... , como elementos imprescindibles para el relanzamiento económico). Directamente relacionado con dicho Programa y como resultado del mismo, se promulgó el Decreto-Ley 1/1992 -más conocido como el "Decretazo"- de Medidas Urgentes de Fomento del Empleo y de Protección por Desempleo, que, entre otras medidas, introdujo la subvención a las contrataciones por tiempo indefinido, la reducción de los costes de protección por desempleo mediante la elevación de los requisitos para causar derecho a estas prestaciones o la intro-ducción de la duración mínima de un año para los contratos temporales de fomen-to de empleo (el RDL 3/93 amplio su duración a un máximo de cuatro años).

Sin descender a más detalle, puede afirmarse que el proceso de reformas ha sido anárquico y poco sistemático y que, desde luego, dichas medidas flexibilizadoras no han frenado la caída de las tasas de ocupación. Las últimas reformas legislativas son, desde el punto de vista de la filosofía que las inspira, continuación de esta tendencia, si bien se trata de una reforma más global, sistemática y programada cuyo eje central es el mercado como elemento regulador (el libre juego de oferta y demanda de empleo y de trabajo y salario), y no la intervención estatal. 
A lo largo de este proceso ha estado latente la idea de atribuir a la nomativa laboral la responsabilidad directa de la actual situación económica y del empleo, sobre todo el incremento constante de los salarios como factor que impide la competitividad de las empresas y el elevado coste de los despidos como factor que obliga a las empresas a la contratación temporal provocando una profunda segmentación del mercado laboral. Sin embargo, resulta difícil admitir que el marco institucional de las relaciones de trabajo tenga una influencia tan decisiva en el desenvolvimiento del sistema económico, siendo su papel mucho más modesto. De otra parte, confluyen demasiadas hipotesis no contrastadas: primero, que la normativa laboral es rígida; segundo, que la rigidez es la causa determinante del desempleo, y tercero, que la supresión de la rigidez provocará la creación de empleo.

No cabe duda de que el marco jurídico-laboral incide sobre el sistema económico -lo cual no equivale a afirmar que sea el origen de sus disfuncionescumpliendo aquél desde sus orígenes un papel instrumental del sistema de producción capitalista. De otro lado, no es fácil aproximar idea sobre el grado de rigidez-flexibilidad de nuestras nomas (2), no siendo posible responder a esta cuestión por comparación, ni tampoco asépticamente. En algunos sentidos se detectan factores comúnmente calificados como de rígidos, tales como la nece-sidad de autorización administrativa previa para los despidos colectivos; sin embargo, para llegar a una visión más exacta se ha de realizar un análisis más global, que, en cualquier caso, estaría mediatizado por la perspectiva políticojurídica de que se parta.

En el análisis del nuevo marco institucional de las relaciones laborales nos limitaremos al estudio de uno sólo de los polos de la reforma, dejando de lado la modificación de las relaciones colectivas y del papel de la negociación colectiva. Para ello y como esquema expositivo analizaremos los diferentes mecanismos de flexibilidad en el desarrollo de la relación de trabajo.

Así nos referiremos, en primer lugar, a los mecanismos de flexibilidad en la entrada al mercado de trabajo: régimen de colocación (donde nos referiremos

(2) Por flexibilidad entendemos, tal como ha definido RODRÍGUEZ-PIÑERO, "el margen de disponibilidad y decisión $y$, por ello a los mecanismos que tiene a su disposición el empresario para enfrentarse a las variaciones cualitativas y cuantitativas de la demanda para reducir el efecto de tales fluctuaciones sobre su estructura de costes", vid. RODRIGUEZPINERO, M. (1993), Relaciones Laborales, 13. 
a las empresas de trabajo temporal y a las agencias privadas de colocacion) y modalidades de contratación (contratos de aprendizaje, en prácticas y a tiempo parcial).

En segundo lugar, estudiaremos los mecanismos de flexibilidad en el desarrollo de la relación laboral: tiempos de trabajo, movilidad funcional, movilidad geográfica, ordenación del salario y modificación de las condiciones de trabajo.

Finalmente, se hara mención de los mecanismos de salida, que flexibilizan la extinción de los contratos, y del coste de los mismo y su agilización, refiriéndonos especialmente a la tan debatida autorización administrativa en los despidos colectivos.

Es evidente que, dadas las características globalizadoras de este trabajo, no descenderemos a pormenorizar el régimen jurídico concreto de cada una de estas instituciones, limitándonos a realizar un estudio selectivo y de carácter general.

\section{Flexibilidad de entrada: el régimen de colocación y las modalidades de contratación}

Uno de los aspectos mediante los que se pretende flexibilizar el mercado de trabajo es la modificación del régimen jurídico de colocación, acometido mediante el Decreto-Ley 18/1993, de 3 de diciembre, de Medidas Urgentes de Fomento de la Ocupación. El aspecto más destacado en esta materia es la consagración legal de las agencias privadas de colocación, rompiendo con nuestra tradición normativa de atribuir a un ente público el monopolio en materia de colocación. Así, aun manteniéndose la función del INEM de "ayudar a los trabajadores a encontrar un empleo y a las empresas a contratar a los trabajadores apropiados a sus necesidades" -art. 43.b) de la Ley Básica de Empleo-, queda derogado por el DL el art. 42.1 de la LBE que en su primer inciso imponía a las empresas la obligación de solicitar previamente en las oficinas de empleo los trabajadores que necesiten; por tanto, se abre la posibilidad por un lado a la contratación directa por el empresario haciendo o no previa publicidad de la oferta de empleo ( $y$ en el primer caso sin el previo visado del INEM) y a la contratación a través de las agencias privadas de colocación no lucrativas.

Hasta el momento el monopolio estatal en materia de colocación se inspiraba en una línea de política intervencionista que, dejando a salvo la libertad empresarial de contratar al trabajador que desease, trataba de garantizar principios 
comóel de igualdad y no discriminación difícilmente salvables con la supresión de todo control y con la vigencia plena de la libre oferta y demanda. El resultado de esta reforma es una ampliación de la libertad empresarial a la hora de elegir la vía para la contratación, así como la disminucion de los controles estatales.

Interesa hacer especial mención, siquiera sea brevemente, a las agencias privadas de colocación. El RDL 18/93 deroga expresamente el art. 16.2 del Estatuto de los Trabajadores, quedando prohibidas tan solo las agencias privadas con fines lucrativos: lo que es lo mismo, se admite la existencia de agencias de colocación que no tengan dicha finalidad lucrativa.

Ciertamente, puede resultar chocante compaginar una actividad privada con un fin no lucrativo. La explicación de esta cuestión ha de ponerse en relacion con el Convenio núm. 96 de la OIT donde se distingue precisamente entre unas y otras, definiendo a las que tienen fin lucrativo como aquéllas que pretenden obtener del trabajador o empresario "un beneficio material directo o indirecto" y a las que no tienen fin lucrativo, si bien pueden percibir del empresario o del trabajador "un derecho de entrada, una cotización o una remuneración cualquiera" por los servicios prestados "cuya cuantía es fijada o aprobada por la autoridad comperente habida cuenta de los gastos ocasionados". En esta línea el RDL dispone que las agencias sin fin de lucro habrán de ser previamente autorizadas por el Servicio público de empleo según el correspondiente convenio de colaboración y, en cualquier caso, "siempre que la remuneración que reciban del empresario o del trabajador se limite exclusivamente a los gastos ocasionados por los servicios prestados" (art. 1.2 RDL).

Se trata de una regulación bastante imprecisa y atípica ya que no quedan determinados aspectos tan importantes como si la retribucion se producirá sólo si se produce efectivamente la colocación o por el mero hecho de la entrada. Igualmente es atípico remitir en bloque a los convenios de colaboración las exigencias a que hayan de someterse las agencias, dado lo parco del RDL y el carácter, en todo caso, reglado de tal competencia administrativa. De otra parte, se echan de menos mecanismos de control que garanticen suficientemente la vigencia de los principios de igualdad y no discriminación o, por decirlo gráficamente, la compraventa de puestos de trabajo.

Directamente relacionado con el régimen de colocación el RDL 18/1993 introduce otra importante novedad en nuestro país: la admisión de las empresas de trabajo temporal, ampliamente conocidas. La LET venía prohibiendo el 
reclutamiento de trabajadores y la contratación de trabajadores para prestarlos o cederlos "cualesquiera que sean los títulos de dicho tráfico de mano de obra" -art. 43-. Lógicamente la admisión de las agencias privadas de colocación implica la supresión de la prohibición de mediación en el mercado de trabajo, siempre que el reclutamiento de trabajadores se produzca en el seno de una agencia debidamente autorizada. Junto a ello, el RDL modifica la segunda de las prohibiciones, la interposición, admitiendo la contratación para prestar o ceder síempre que la misma se produzca en el seno de una empresa de trabajo temporal (art. 2.1 del RDL).

Igual que en lo relativo a las agencias privadas, se tratade una técnica legislativa criticable en cuanto que no parece justificado acudir a un instrumento como el Decreto Ley, que por definición presupone la nota de urgencia, y porotro remitirse en su regulación a lo que legalmente se establezca. EI RDL parece que legaliza estas empresas, definitivamente reguladas porla Ley 14/1994. Estas empresas han de contar con la correspondiente autorización administrativa, la cesión es temporal, ya que el vínculo jurídico-laboral se establece con la propia empresa de trabajo temporal (ETT) y no con el empresario que percibe dicha prestación temporal. Se regula el contrato de puesta a disposición, el régimen de responsabilidad entre la empresa usuaria y la ETT, y el contrato de trabajo en la ETT.

Dentro de lo que hemos dado en llamar mecanismos de flexibilizacion en la entrada se enmarcan las modalidades de contratación, las cuales también se han visto afectadas por la reforma: concretamente, los contratos en prácticas, los contratos para la formacion -que desaparecen, ocupando su lugar los contratos de aprendizaje- y los contratos a tiempo parcial.

Los contratos formativos -tanto el contrato para la formación como el contrato en prácticas-, aun concebidos inicialmente con una finalidad formativa, en su evolución han sido orientados en las sucesivas reformas normativas como instrumentos de la política de fomento de empleo, en detrimento de su función originaria. En este sentido la reforma producida por el RDL 18/1993 de 3 de diciembre -desarrollado por el RD 2317/93, de 29 de diciembre- supone, en cierta manera, una inflexión en la línea señalada. No obstante, se trata de una inflexión parcial ya que tan sólo afecta a los contratos en prácticas.

Estos, como contratos de duración determinada orientados a la adquisición de una práctica que complete la formación térica adquirida en centros educativos, se reorientan hacia su sentido originario garantizando su papel 
esencialmente formativo frente al componente de fomento de empleo que pasa a ocupar ahora un papel secundario.

Muy al contrario, los contratos para la formación desaparecen abriendo paso a una nueva modalidad: el contrato de aprendizaje, que pretende ser una modalidad formativa para la mano de obra juvenil no cualificada. Paradojicamente, su regulacion apenas presta atencion a los aspectos capacitadores: por el contrario, se trata de una modalidad que prima las exigencias de empleo a toda costa; concretamente se prevé un abaratamiento del coste salarial y de los costes de seguridad social, con la consiguiente reducción de la cobertura social.

En el caso del contrato de aprendizaje se ha acudido al argumento de la recuperación de una figura historica, al argumento de los modelos comparados, tales como el alemán o el francés, dejando en la sombra la verdadera función economico-social de estos contratos, que no es otra que proporcionar mano de obra barata.

Pudiera pensarse que la reforma, al menos, salvaguarda la vertiente formativa mediante la figura de los contratos en prácticas. Sin embargo, la existencia misma de los contratos de aprendizaje pone en peligro la de aquéllos desde el punto que, en base a la libertad contractual y de trabajo, resulta difícil excluir a los titulados del ámbito de los contratos de aprendizaje, y ello en un momento en que la demanda de empleo es con mucho superior a la oferta.

Finalmente, otra de las modalidades de contratación afectada por la reforma es el contrato a tiempo parcial, donde también se manifiestan las opciones flexibilizadoras y de abaratamiento de los costes sociales. El mismo queda definido -art. 4 RDL 18/1993- en términos más amplios que en la regulación anterior, de modo que bastará la contratación de servicios durante un número de horas semanales, mensuales o anuales "inferior al considerado como habitual", en lugar de "inferior a dos tercios" que fijaba el art. 12 de la LET. Ello se aparta de los criterios de la OIT, que considera contrato a tiempo parcial aquél cuya jornada es sensiblemente inferior a la normal, y facilita el uso de esta modalidad contractual. De otro lado, la introducción del año como parámetro de referencia impide diferenciar el contrato para trabajos fijos discontinuos de carácter periódico del contrato a tiempo parcial, pasando aquél a ser una modalidad de este, lo que introduce notables disfuncionalidades. Sin embargo, la novedad más importante se refiere al terreno de la seguridad social, ya que la base de cotización pasa a estar constituida por las retribuciones percibidas en función del número de horas trabajadas (art. 4.3 RDL) y no en función de los días, lo que, 
como es evidente, empeora la situación de estos trabajadores y abarata los costes sociales. Adicionalmente, el régimen de protección se ve sensiblemente reducido en determinados contratos a tiempo parcial: aquéllos "cuya prestación efectiva de servicios sea inferior a doce horas a la semana o cuarenta y ocho al mes", en cuyo caso los derechos de protección social sólo incluirán las contingencias de accidentes de trabajo y enfermedades profesionales, asistencia sanitaria por contingencias comunes y Fondo de Garantía Salarial. Por tanto, quedan excluidos de la protección por incapacidad laboral transitoria, invalidez provisional, pensiones y desempleo.

\section{Flexibilidad en el desarrollo y extinción de la relación laboral}

Al igual que en el apartado anterior, no se realiza un análisis propiamente dicho del régimen jurídico de cada una de las instituciones afectadas, limitándonos a dar una visión más de conjunto.

Los mecanismos flexibilizadores y reindividualizadores que la Ley 11/1994 introduce en la regulación del contrato de trabajo tienen especial incidencia en la fase de desarrollo de la relación laboral, afectando tanto al tiempo de trabajo, como a la determinación espacial de la prestación de servicios (movilidad geografica), a la determinación objetiva de la misma (movilidad funcional), a los salarios y a los límites del poder de dirección empresarial (modificación sustancial de las condiciones de trabajo).

Tal como queda expresado en la propia Exposición de Motivos de la Ley, "es quizá en el desarrollo de las relaciones laborales donde la necesidad de flexibilidad se hace más patente, posibilitando una mayor adaptabilidad de los elementos definidores de la relación laboral -en cuanto al contenido, el lugar o el tiempo de la misma, así como las restantes condiciones de trabajo- a las necesidades cambiantes de los mercados y de los procesos productivos. No hay duda pues del sentido político-jurídico de la reforma que, sin ambages de ningún tipo, procede a un cambio profundo no sólo del contenido de determinados preceptos sino de la fuente de regulación; es decir, que lo que hasta ahora se han considerado verdaderos mínimos inderogables desaparecen -desregulación-, remitiéndose su ordenación ya a la autonomía colectiva (a los convenios colectivos), en unos casos, ya a la autonomía individual (al contrato de trabajo), en otros. 
El resultado de estos cambios que, aparentemente, solo interesan a los teóricos dedicados al estudio de las relaciones de trabajo, trasciende las propias relaciones laborales afectando a las formaciones sociales de una forma más amplia. Pensemos que la precariedad salarial, la temporalidad, la inestabilidad en el lugar de la prestación, en el horario, en la jomada, en las funciones a desempeñar, en la categoría... pueden incidir en los modos de vida, en la tasa de natalidad, en la dimensión de las viviendas, en las formas familiares tradicionales..., porponer algunos ejemplos. Sin llegar a plantearlo en términos proféticos, digamos que la profunda modificación del trabajo asalariado, de su régimen jurídico, afecta a una gran parte de la población cuyo medio de vida es el intercambio de su trabajo por salario, lo cual verá alterada sus formas de vida.

La ordenación de los tiempos de trabajo -jomada, horario, vacaciones, descansos...- constituye un elemento básico del régimen jurídico-laboral, donde confluyen intereses privados de empresarios (pues es un elemento fundamental de la organización empresarial con gran incidencia en la eficiencia del sistema productivo) y de trabajadores, así como intereses públicos (fundamentalmente, la protección de la salud y de la seguridad de los trabajadores y la política de empleo, ya que no olvidemos que la limitación de la jomada y el reparto de trabajo, son una de las líneas de la política de fomento de empleo).

Los artículos de la LET referidos a la ordenación de los tiempos de trabajo reformados por la Ley 11/94 (arts. 34, 35, 36 y 37) afectan, en primer lugar, a la regulación de la jornada y, concretamente, a los topes máximos, ya que la duración máxima de cuarenta horas semanales de trabajo efectivo ya no tiene como módulo de referencia la semana sino el año, de modo que el límite semanal desaparece. Parece que la superación de las cuarenta horas semanales, aunque respete el tope en cómputo anual, quedaría reservado a la autonomía colectiva ya que el nuevo artículo 35 dispone que por convenio colectivo o, en su defecto, por acuerdo entre la empresa y los representantes de los trabajadores podrá pactarse una distribución irregular de la jornada a lo largo del año, siempre que se respeten el descanso diario y el descanso semanal. No obstante, no parece pueda excluirse terminantemente que mediante acuerdo interpartes se pacten jornadas que en cómputo semanal superen las cuarenta horas (aunque desde luego juega el tope de nueve horas ordinarias diarias), ya que el precepto cambia del modulo de referencia con carácter general. 
Más importante es destacar el cambio de tendencia en la regulación de las horas extraordinarias, cuyo tratamiento normativo, hasta ahora, era claramente "penalizador": en este sentido se establecía un incremento salarial mínimo, respecto del coste de la hora ordinaria (de un $75 \%$ ), con la finalidad de proteger la salud de los trabajadores de jornadas extenuantes con mayor riesgo de accidente laboral y de fomentar el empleo incentivando el reparto del empleo disponible. La nueva regulación sólo garantiza que se pague al menos como las horas ordinarias, lo que en teoría desincentivará su realización; esta interpretación vendría, además, confirmada por el propio precepto al disponer que, en defecto de pacto, la opción entre el abono de estas horas o su compensación con tiempos de descanso se presumirá hecha en favor del descanso. Sin embargo, la realidad es que el abaratamiento de estas horas -salvo que los convenios dispongan lo contrario-, mucho más interesantes para las empresas que acudir a nuevas contrataciones a tiempo parcial, incentiva a las empresas a su utilización; y, aunque su realización es voluntaria para los trabajadores, el poder de negociación de éstos en el plano individual es inexistente. Por tanto, opino que la reforma no incidirá en el reparto de empleo y, por el contrario, afectará al derecho al descanso, una de las más viejas reivindicaciones de la lucha obrera.

Aunque también se ven afectadas otras instituciones, tales como el trabajo noctumo oel trabajo a turnos, no podemos, en un trabajo de estas características, entrar a tal nivel de detalle, limitándonos a ilustrar las líneas maestras de la reforma.

Otro aspecto en el que se plasma la política de flexibilización es el referido a la movilidad geografica, afectando especialmente al régimen jurídico de los traslados. El art. 40 reformado mantiene el principio de causalidad, exigiendo que el mismo se base en razones objetivas; sin embargo, se amplía el elenco de éstas pues, junto a las razones técnicas, organizativas o productivas, se introducen las razones económicas. Y, lo que es más importante, adicionalmente se modifica el mecanismo de control de objetividad de las decisiones empresariales de traslado al suprimirse la necesidad de autorización administrativa previa. La orden de traslado pasa a tener ejecutividad inmediata y, aunque en el caso de los traslados colectivos existe un período de consultas y obligación de negociar con los representantes de los trabajadores, el trabajador ha de someterse a la decisión empresarial o extinguir su contrato. Una vez trasladado, el trabajador podrá impugnar la decisión ante la jurisdicción competente, la cual declarará si el trastado ha sido o no justificado. 
Desde el punto de vista empresarial se trata de un cambio que amplía sus facultades directivas, modificando unilateralmente el lugar de la prestación pactado, cosa que puede redundar en una gestion más eficiente y ágil. En cambio, del lado del trabajador, se acentúa la nota de subordinación -con graves repercusiones en su vida privada- y el riesgo de sufrir discriminaciones arbitrarias o despidos encubiertos.

Un cambio semejante sufre el procedimiento de modificación sustancial de las condiciones de trabajo previsto en el art. 41 LET. Al respecto la Exposicion de Motivos de la Ley 11/94 señala que la modificación pretende establecer" "las necesarias garantías de los derechos de los trabajadores frente a decisiones, a través de un adecuado control de la justificación de la medida, una participación más intensa de los representantes de los trabajadores e incluso, en determinadas ocasiones, el reconocimiento del derecho de los trabajadores perjudicados por ellas a rescindir su contrato de trabajo. Sin embargo, dichas garantías no pueden llegar a dificultar hasta el extremo de imposibilitarla adopción de las medidas que resulten necesarias, pues con ello, lejos de proteger a los trabajadores, se pondría en peligro su empleo y la viabilidad de la empresa en su conjunto. La nueva regulación de los artículos 40 y 41 en materia de movilidad geografica y modificación sustancial de las condiciones tiene en cuenta estos principios estableciendo un procedimiento ágil basado en los principios de causalidad y el control judicial así como en la participación de los organos colectivos en las decisiones de tal naturaleza". Como puede observarse el cambio del régimen de estas modificaciones sustanciales reside, fundamentalmente, en los mecanismos de control, que pasan de la negociación con los representantes de los trabajadores -hasta ahora la falta de acuerdo entre estos y el empresario impedía a éste realizar cualquier modificación sustancial- a la mera consulta (y soblo en el caso de que se trate de una modificación de carácter colectiva) y de la necesidad de autorización administrativa previa-que, a falta de acuerdo, era necesaria- al control judicial, que necesariamente es un control a posteriori, una vez se han producido las modificaciones. De este modo, el empresario puede proceder, siempre que se ajuste al principio de causalidad, a modificar unilateralmente, entre otros, la jomada de trabajo, el horario, sistema de trabajo a turnos, sistema de remuneración, sistema de trabajo y rendimiento o el cambio de funciones más allá de los límites del art. 39. Por tanto, la reforma viene a reforzar el poder de dirección empresarial, potenciando las facultades del empresario al que se le reconoce una verdadera 
capacidad decisoria con efectos ejecutivos e, implícitamente, parte de considerar que la negociación colectiva y la autorizacion administrativa han venido impidiendo la adopción de medidas necesarias para la viabilidad de las empresas.

La determinación objetiva de la prestación de servicios sufre, igualmente, modificaciones importantes dirigidas a procurar una gestion empresarial más eficiente de los recursos humanos. Uno de los ejes centrales de la reforma es posibilitar una definición más flexible, desde el ingreso al trabajo, del contenido de la prestación laboral pactada, superando los rígidos esquemas de la clasificación profesional y ampliando la flexibilidad para adaptar dicha prestación a la variación de las necesidades empresariales. Esto se instrumenta mediante la noción de polivalencia funcional y el nuevo procedimiento de modificación sustancial de las condiciones de trabajo que rompen con el derecho subjetivo a la categoría.

Otro de los caballos de batalla emprendidos por la Ley $11 / 1994$ se refiere a los mecanismos de flexibilidad de salida, o, dicho en otros términos, a los mecanismos extintivos de la relación laboral y, especialmente, los despidos disciplinarios y los despidos económicos.

Del conjunto de disposiciones ordenadoras del mercado de trabajo, las que afectan a la extinción del contrato por causas económicas y, concretamente, la función que en estos despidos haya de desempeñar la Administración constituyen lo que podríamos denominar núcleo duro del debate, habiéndose acudido a argumentos justificadores de la supresión de la autorización poco precisos o mixtificadores, tales como el derecho comparado o la necesidad de adecuamos a la normativa comunitaria. La LET establecía la necesidad, en todo caso, de negociación con los representantes de los trabajadores y, una vez agotada ésta sin acuerdo entre el empresario y el banco social, la intervencion de la Administración.

La necesidad de autorización administrativa en estos despidos, a diferencia del resto (sometidos a control judicial a posteriori), se funda en la trascendencia social de los mismos dado el impacto que produce el despido de un número importante de trabajadores. Junto a la trascendencia social, se enfrentan aquí intereses de los trabajadores, para quienes el sistema de autorización resulta más garantista del principio de estabilidad en el empleo (y en su defecto una adecuada compensación económica), y los de los empresarios, para los que este sistema incidiría negativamente en la gestión ágil y flexible de la empresa y en su poder de negociación frente a los trabajadores. 
El conflicto de intereses subyacente se ha resuelto, finalmente, y tras muchos titubeos, por una especie de solución intermedia que suprime la autorización en el caso de que el despido económico afecte a un número reducido de trabajadores, manteniéndola para el resto.

Finalmente, cabe destacar, entre las modificaciones referidas a los mecanismos extintivos y dirigidas a lograr una mayor flexibilidad de salida, las que afectan al despido disciplinario.

Sin entrar en un análisis pormenorizado, la modificación más importante afecta a las distintas categorías en que se pueden clasificar estos despidos (procedente, improcedente o nulo). El despido nulo, por defecto de forma o de fondo, tenía como efecto la readmisión obligatoria para el trabajador; no obstante, en la práctica, la reanudación no era frecuente, sustanciándose el contencioso mediante el pago de indemnización más elevada que la prevista legalmente para el caso de los despidos declarados improcedentes. La Ley 11/94 califica el despido en que no se hayan observado los requisitos formales de improcedente y nulo solamente cuando el despido tenga carácter discriminatorio o se base en una causa prohibida por la Constitución o por la ley, o bien se produzca con violación de derechos fundamentales y libertades públicas del trabajador. Se elimina, por tanto, como causa de nulidad el despido durante la situación de suspensión del contrato.

De otro lado, en materia de recursos contra las sentencias de despido, se establecía la obligación empresarial de pago de salarios mientras se sustanciaba el correspondiente recurso (lo cual frenaba la interposicion de recursos del lado empresarial). La reforma elimina dicha obligación, de modo que la opción del empresario, en el caso de los despidos improcedentes, por la indemnización le exoneraría de la readmisión provisional y, en todo caso, (se incorpore o no dicho trabajador) de las remuneraciones correspondientes.

Así, y a modo de conclusión, la reforma incide en un cierto abaratamiento de estos despidos libres aunque indemnizados.

A modo de colofón final, puede señalarse que se trata de una reforma de signo flexibilizador, probablemente inconclusa, a la espera de la complementaria reforma del sistema de seguridad social y cuyo resultado final proyectará sus efectos más allá del campo de las relaciones laborales. 